\title{
A quantitative evaluation of the determinant proteins for $S-1$ responsiveness in a biopsy specimen assists in patient selection to neoadjuvant therapy in cases of advanced gastric cancer
}

\author{
KAZUYOSHI HASHIGUCHI ${ }^{1}$, YOSHIHIKO KITAJIMA ${ }^{1}$, KEITA KAI $^{1,2}$, MASATSUGU HIRAKI $^{1}$, \\ JUN NAKAMURA $^{1}$, OSAMU TOKUNAGA ${ }^{2}$, HIROKAZU NOSHIRO ${ }^{1}$ and KOHJI MIYAZAKI ${ }^{1}$ \\ Departments of ${ }^{1}$ Surgery and ${ }^{2}$ Pathology and Biodefense, Saga University Faculty of Medicine, Saga, Japan
}

Received February 19, 2010; Accepted April 20, 2010

DOI: 10.3892/ijo_00000673

\begin{abstract}
Neoadjuvant chemotherapy (NAC) by 5 -fluorouracil including S-1 is administered to advanced gastric cancer patients. However, the therapeutic benefit from this preoperative treatment remains uncertain. The present study analyzed the expression of 5-fluorouracil related enzymes, TS, DPD and OPRT in 47 gastric cancer biopsy specimens using quantitative double-fluorescence immunohistochemistry (qDFIHC), which is a newly developed system to quantify protein expression. The study first determined whether the cancer heterogeneity within the sample influences evaluation by qDFIHC system. Thereafter, the expression values of the TS, DPD, OPRT and OPRT/TS, OPRT/DPD, OPRT/ (TS+DPD) ratios were retrospectively correlated with the clinical or pathological response in the patients. The expression values of TS, DPD and OPRT at a single field were significantly correlated with mean of the values evaluated at three fields. Among the 6 candidate factors analyzed, OPRT, OPRT/TS, OPRT/DPD and OPRT/(TS+DPD) showed significant correlations with the clinical response in 47 patients. Cut-off values to differentiate the clinical response were determined in the four factors. OPRT/TS showed the strongest correlation with the clinical response. qDFIHC was able to quantify the TS, DPD and OPRT expressions in cancer biopsy specimens without being affected by the heterogeneity. Effective therapy using tailored S-1 NAC according to the OPRT/TS ratio is therefore expected in advanced gastric cancer patients.
\end{abstract}

Correspondence to: Dr Yoshihiko Kitajima, Department of Surgery, Saga University Faculty of Medicine, 5-1-1 Nabeshima, Saga 849-8501, Japan

E-mail: kitajiy@cc.saga-u.ac.jp

Key words: S-1, tailor-made chemotherapy, qDFIHC, gastric cancer

\section{Introduction}

Gastric cancer remains one of the leading causes of cancerrelated deaths in the world (1). 5-fluorouracil (5-FU) is a commonly used agent for the treatment of gastric cancer. S-1 is an advanced type of 5-FU and composed of an oral formulation of tegafur (FT) which is a prodrug of 5-FU, 5-chloro-2,4dihydroxypyridine (CDHP) which is an inhibitor of dihydropyrimidine dehydrogenase (DPD) and potassium oxonate $(\mathrm{OXO})$ which is a protective agent against FT-induced diarrhea $(2,3)$. At present, S-1 is accepted as the key component of the standard chemotherapy for advanced gastric cancer in Japan. However, the response rate (RR) to S-1 based chemotherapy is $\sim 30 \%(4,5)$. Neoadjuvant chemotherapy (NAC) by S-1 is also administered to patients with resectable gastric cancer whose prognosis is considered to be poor, even after undergoing a surgical resection $(6,7)$. However, whether NAC contributes to prolonging survival with advanced gastric cancer still remains unknown. If NAC fails to reduce the tumor spread, then this preoperative therapy might not only provide a harmful effect to the patients, but it may also result in a decision to not perform subsequent needed surgery. The identification of a reliable marker for predicting S-1 responsiveness is therefore urgently needed to solve the problems in NAC.

The mechanism of cytotoxicity for 5-FU causes misincorporation of fluoronucleotides into RNA and DNA. First, 5-FU is activated by orotate phosphoribosyl-transferase (OPRT) and then the metabolite is activated several times. Finally, 5-FU is converted to fluorodeoxyuridine monophosphate (FdUMP). FdUMP forms an irreversible complex with the nucleotide synthetic enzyme thymidylate synthase (TS). This complex inactivates the TS function, thus leading to an impaired DNA synthesis. On the other hand, most administered 5-FU is broken down by DPD (8). Therefore, the expression of TS, DPD or OPRT in cancer cells has been highlighted as candidate molecules for predicting 5-FU efficacy. Theoretically, cancer cells with lower TS, lower DPD and higher OPRT activities are more sensitive to 5-FU than those with a higher TS, higher DPD and lower OPRT. There is a significant relationship between the 
expression of these enzymes and 5-FU responsiveness (9-13). TS, DPD and OPRT expressions have been quantitatively assessed by such procedures as real-time RT-PCR (14-18) and an enzyme-linked immunosorbent assay (ELISA) $(18,19)$ using surgically resected colorectal and gastric cancer specimens. The resultant findings demonstrated a high TS, high DPD or low OPRT expression in tumor tissue to be correlated with resistance against 5-FU (9-13). A recent study compared the enzyme activity of DPD with the protein expression assessed by ELISA, IHC or mRNA expression by RT-PCR using lung cancer tissues (20). The results showed the highest correlation to be observed between the protein expression measured by ELISA and the enzyme activity. The correlation between the gene expression by RT-PCR and that by ELISA was also significant, thus suggesting ELISA and RT-PCR to be suitable for the quantitative measurement of 5-FU related enzymes (20). However, either ELISA or real-time RT-PCR requires fresh frozen tissue specimens, and sometimes a micro-dissection step to isolate cancer cells. Therefore, these procedures might be difficult to quantify either the gene or protein expression in tiny cancer tissue specimens obtained from an endoscopic biopsy. On the other hand, immunohistochemistry (IHC) is the most common method to analyze protein expression even in formalin-fixed biopsy specimens, however this conventional method might not be effective for quantitative assessments.

A quantitative double-fluorescence immunohistochemistry (qDFIHC) system was developed in 2007 and one study attempted to quantify the expression of TS, DPD and OPRT in 17 formalin-fixed cancer biopsy specimens (13). A qDFIHC analysis acquires digital images from each enzyme labeled by FITC and internal marker $\beta$-actin by $\mathrm{Cy} 3$ on a confocal Laser Scanning Microscope at magnification $x 400$. The quantification value of TS, DPD or OPRT was represented as the ratio to $\beta$-actin (13). The qDFIHC method can focus on small amount of cancer foci and quantitatively measure the protein expression even in 10 cancer cells. The study demonstrated a significant correlation between OPRT/TS, OPRT/DPD or OPRT/(TS+DPD) and S-1 efficacy (13) in the gastric cancer patients. However, several problems still remain to be solved. First, the qDFIHC study analyzed protein expression only at a single field in a specimen. However, consistent data may not be obtained in an analysis of only a single field because of the heterogeneity within the gastric cancer tissue. Second, the number of patients analyzed was too small for a statistical analysis.

The present study assessed the TS, DPD and OPRT expression levels by the qDFIHC system using 47 gastric cancer biopsy specimens obtained before $\mathrm{S}-1$ treatment. The ratios of OPRT/TS, OPRT/DPD and OPRT/(TS+DPD) were also determined. The expression of the 3 proteins were assessed in three fields and compared with those in a single field, in order to investigate the possible heterogeneity in the same sample. The expression values of the six factors were then correlated with S-1 efficacy in 47 patients to isolate candidates predicting S-1 efficacy. The predictive powers of the candidates were then further evaluated with respect to the sensitivity, specificity and accuracy. The current study discusses the feasibility of S-1 prediction by a qDFIHC system to tailored NAC in advanced gastric cancer patients.

\section{Materials and methods}

Clinical method. Forty-seven patients with advanced gastric cancer, who underwent S-1 based chemotherapy between 2001 and 2009 at the Department of Surgery, Saga University Hospital (Saga, Japan), were enrolled in this study. All of the patients provided their written informed consent approved by the Ethics Committee. The stage classification was determined according to the UICC TNM classification (21). Forty-three patients had advanced carcinoma with a clinical stage higher than stage III. Four patients who were classified as stage IB were preoperatively treated by $\mathrm{S}-1$ because the clinical diagnosis was scirrhous type gastric cancer. The regimens of the $\mathrm{S}-1$ based chemotherapies were, $\mathrm{S}-1$ alone $(\mathrm{n}=6), \mathrm{S}-1$ plus cisplatin $(n=29)$ and $S-1$ plus paclitaxel $(n=12)$. One cycle of each regimen was: i) the $\mathrm{S}-1$-alone regimen was the oral administration of S-1 (Taiho Pharmaceutical Co., Tokyo, Japan) at $80 \mathrm{mg} / \mathrm{m}^{2} /$ day (22), which was continued for 28 days and followed by a rest period of 14 days (2). In the S-1 plus cisplatin regimen, $\mathrm{S}-1$ was administered in the same manner as the $\mathrm{S}$-1-alone regimen and the $5-20 \mathrm{mg} /$ body of cisplatin was intravenously administered at days 1,8 and 15 (3). In the S-1 plus paclitaxel regimen, S-1 was administered for 2 weeks, and $80 \mathrm{mg} / \mathrm{m}^{2}$ paclitaxel was intravenously administered on days 1 and 15 . The mean cycle number of the therapeutic regimens was 3.47 cycles (ranging from 1 to 16 cycles). After the S-1 based chemotherapy, 17 of the 47 patients underwent a gastrectomy with a regional lymph node dissection.

Evaluation of effect of chemotherapy. The antitumor effects and the adverse reactions were evaluated in accordance with the Japanese Classification of Gastric carcinoma, 2nd English edition (23), which was established based on the WHO criteria. The criteria for the evaluation of antitumor effects were: a complete response (CR), a partial response (PR), no change (NC), and progressive disease (PD). The patient groups were further divided into responder $(\mathrm{CR}+\mathrm{PR})$ and non-responder $(\mathrm{NC}+\mathrm{PD})$ groups (23). The response rate (RR) was conventionally calculated as the ratio of responder/responder + non-responder. The pathological response was determined according to the amount of necrosis or the disappearance of the tumor cells in the estimated total amount of the lesion, which was also graded into five categories, Grade $0,1 \mathrm{a}, 1 \mathrm{~b}$, 2 and 3, according to the criteria of the Japanese Research Society for Gastric Cancer (24). Grade 3 was assessed as pathological CR (pCR), Grade 1b and Grade 2 were as pPR, Grade 0 and Grade 1a were as pNC and clinical PD was also regarded as $\mathrm{pNC}$.

Double-fluorescence immunohistochemistry (IHC). The formalin-fixed paraffin-embedded gastric biopsy samples obtained before chemotherapy were cut into $4 \mu \mathrm{m}$ sections. The sections were deparaffinized in xylol and dehydrated in a graded alcohol series. The primary antibodies, TS (rabbit polyclonal, dilution 1:100), DPD (rabbit polyclonal, dilution 1:100) and OPRT (rabbit polyclonal, dilution 1:500) were provided by the Taiho Pharmaceutical Co., Tokushima, Japan. The secondary antibody for TS, DPD and OPRT was Fluorescein isothiocyanate isomer 1 (FITC) conjugated 
Table I. Comparison of clinicopathologic background of responder and non-responder.

\begin{tabular}{|c|c|c|c|}
\hline & $\mathrm{R}$ & NR & P-value \\
\hline $\begin{array}{l}\text { Age } \\
\qquad(30-84)\end{array}$ & $\begin{array}{c}64.3 \pm 12.6 \\
(34-85)\end{array}$ & $61.9 \pm 14.8$ & $0.588^{\mathrm{a}}$ \\
\hline \multicolumn{4}{|l|}{ Gender } \\
\hline Male & 14 & 20 & $0.871^{\mathrm{b}}$ \\
\hline Female & 5 & 8 & \\
\hline \multicolumn{4}{|l|}{ Histology } \\
\hline Differentiated & 5 & 7 & $0.811^{\mathrm{b}}$ \\
\hline Undifferentiated & 14 & 21 & \\
\hline \multicolumn{4}{|l|}{ Stage } \\
\hline I or II & 1 & 3 & $0.638^{\mathrm{b}}$ \\
\hline III or IV & 18 & 25 & \\
\hline \multicolumn{4}{|l|}{ Regimen } \\
\hline S-1(7) & $\mathrm{I} 2$ & 5 & $0.583^{\mathrm{b}}$ \\
\hline $\mathrm{S}-1+\mathrm{CDDP}(28)$ & 13 & 15 & \\
\hline S-1+PTX(12) & 4 & 8 & \\
\hline $\begin{array}{l}\text { Cycle } \\
(1-13)\end{array}$ & $\begin{array}{c}3.37 \pm 2.77 \\
(1-16)\end{array}$ & $3.54 \pm 4.05$ & $0.174^{\mathrm{a}}$ \\
\hline \multicolumn{4}{|l|}{ Gastrectomy } \\
\hline Yes & 7 & 10 & $0.818^{\mathrm{b}}$ \\
\hline No & 12 & 18 & \\
\hline
\end{tabular}

$\mathrm{R}$, responder, NR, non-responder, CDDP, cisplatin, PTX, paclitaxel. ${ }^{\mathrm{a}}$ Mann-Whitney's U test; ${ }^{\mathrm{b}} \chi^{2}$ test.

anti-rabbit immunoglobulin (IgG; DakoCytomation, CA, USA, dilution 1:100). The primary antibody for internal control was $\beta$-actin antibody (mouse monoclonal antibody, Sigma, St. Louis, USA, dilution 1:500) and the secondary antibody for B-actin was Cy3 conjugated secondary antibody (antimouse IgG, Chemicon International Inc., dilution 1:100). The details of the process were almost the same as those published in a previous report (13).

Quantitative evaluation of TS, DPD and OPRT on the LSM system. The digital images were acquired on a confocal Laser Scanning Microscope LSM5 Pascal (Carl Zeiss Microimaging, Jena, Germany) at magnification $\mathrm{x} 400$. The expression values of the TS, DPD and OPRT were estimated in a single field of view in total 47 sections. Those were further assessed in the three fields of view and the average of three fields were calculated in 30 of 47 sections to evaluate the heterogeneity in the same sample. The fluorescent signals of the images were analyzed by the 'LSM Image Examiner' attached software program in the LSM system as previously described (13). The ratio of the total fluorescence intensity of the target proteins to $\beta$-actin (target protein/ $\beta$-actin) was considered to be the quantification value.
Statistical analysis. The statistical analysis was carried out using the SPSS software package (SPSS, Inc., Chicago, IL). The Mann-Whitney's U test, the $\chi^{2}$ test, Fisher's exact test and Spearman's rank correlation coefficient test were appropriately used in each evaluation. The cut-off value was determined based on a Receiver operating characteristic (ROC) curve. A $\mathrm{P}<0.05$ was considered to indicate a statistically significant difference.

\section{Results}

Clinicopathological features. The clinicopathological features of the 47 patients are demonstrated in Table I. The patients were divided into 4 groups according to the clinical evaluation of chemotherapy, CR $(n=4), P R(n=15), N C(n=21)$ and PD $(n=7)$. The overall response rate $(\mathrm{CR}+\mathrm{PR} /$ total cases $)$ was $40.4 \%$ (19/47 cases). There were no significant differences in age, gender, histology, clinical stage, chemotherapeutic regimen, chemotherapy cycles, and with or without the gastrectomy after chemotherapy between the responder $(\mathrm{CR}+\mathrm{PR})$ and non-responder (NC+PD).

The correlation of the protein expression level of a single field to three fields assessed by qDFIHC. The expression of each protein was analyzed at the three fields in 30 of 47 samples to investigate the heterogeneity within cancer tissue. The mean value in the 3 fields was compared with that in a single field. As a result, the expression value in a single field significantly correlated with the average at the three fields: TS $(|\mathrm{R}|=0.831$, $\mathrm{P}<0.001)$, DPD $(|\mathrm{R}|=0.981, \mathrm{p}<0.001)$ and OPRT $(|\mathrm{R}|=0.976$, $\mathrm{P}<0.001$; Fig. 1).

qDFIHC analysis of the responders and non-responders. The relative expression of the 3 proteins was compared between the responders and non-responders (Fig. 2a). The quantification value of each group (mean \pm SD) was: responders; TS $1.18 \pm 0.35$, DPD 1.39 \pm 0.57 , OPRT 1.67 \pm 0.72 , non-responders; TS $1.16 \pm 0.44$, DPD $1.30 \pm 0.61$, OPRT $1.17 \pm 0.58$. OPRT expression showed a significant difference between the responders and non-responders $(\mathrm{P}=0.012)$. The ratios of OPRT/TS, OPRT/DPD, OPRT/(TS+DPD) were next calculated. The mean \pm SD of each ratio was: responders: OPRT/TS $1.45 \pm 0.51, \mathrm{OPRT} / \mathrm{DPD} 1.25 \pm 0.40, \mathrm{OPRT} / \mathrm{TS}+\mathrm{DPD})$ $0.65 \pm 0.18$; non-responders: OPRT/TS $1.06 \pm 0.51$, OPRT/DPD $0.95 \pm 0.26, \mathrm{OPRT} /(\mathrm{DPD}+\mathrm{TS}) 0.48 \pm 0.13$. A significant difference was observed in OPRT/TS $(\mathrm{P}=0.005)$, OPRT/DPD $(\mathrm{P}=0.006)$ and $\mathrm{OPRT} /(\mathrm{TS}+\mathrm{DPD} ; \mathrm{P}=0.002)$.

Cut-off value determined by an ROC curve and an evaluation of the $S-1$ prediction. The optimal cut-off value for OPRT, OPRT/TS, OPRT/DPD, and OPRT/(TS+DPD) was determined according to each ROC curve (Fig. 2b). The cut-off value was 1.2 for OPRT, 1.2 for OPRT/TS, 1.1 for OPRT/DPD and 0.6 for OPRT/(TS+DPD), respectively (Fig. 2c). Based on the cut-off value, the sensitivity, specificity and accuracy to the clinical response was determined and compared among OPRT, OPRT/TS, OPRT/DPD, and OPRT/(TS+DPD; Table II). OPRT/TS exhibited the highest percent in the sensitivity $(73.7 \%)$, specificity $(82.1 \%)$ and accuracy $(78.7 \%)$. The tailored response rate (tRR) was also determined as RR 

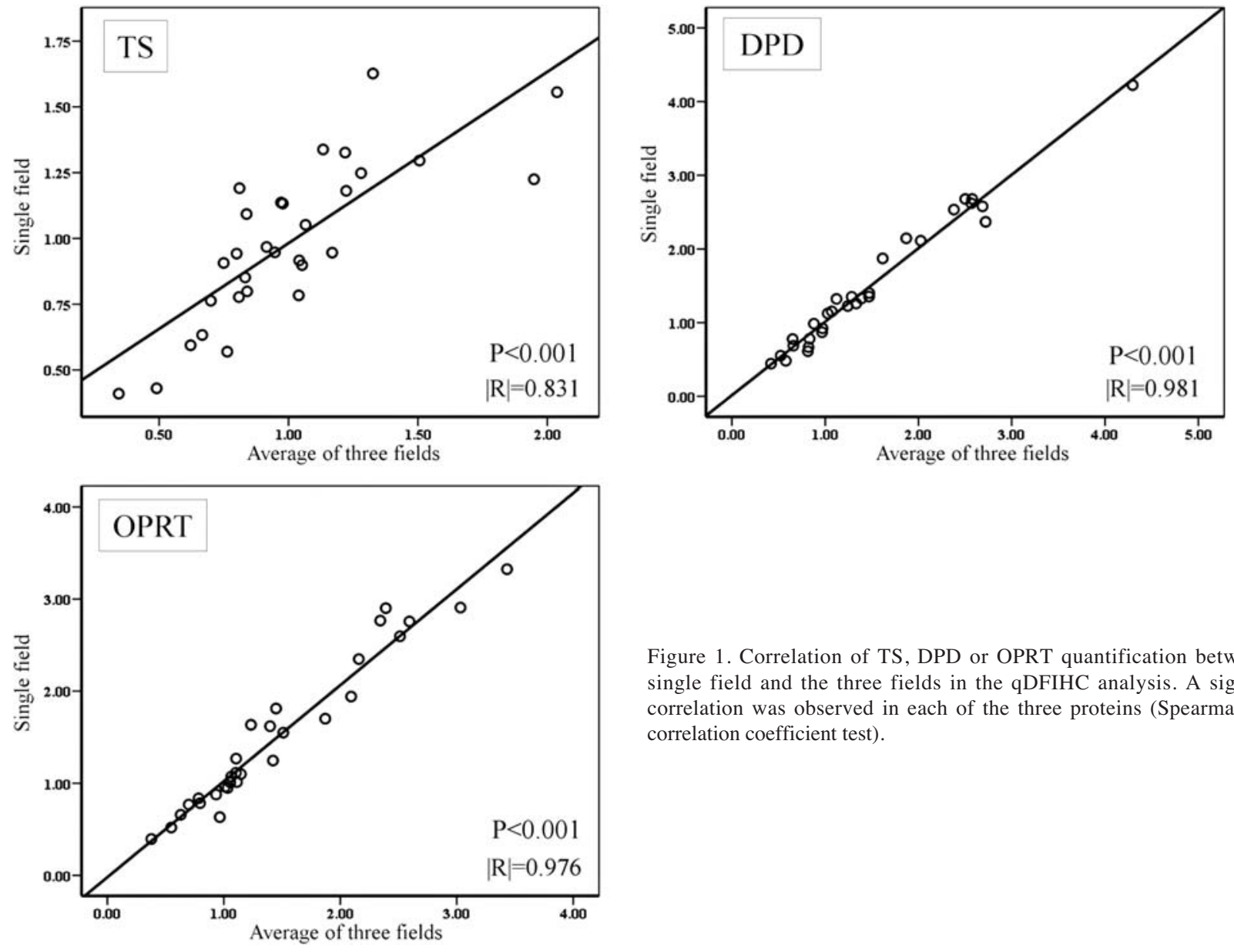

Figure 1. Correlation of TS, DPD or OPRT quantification between the single field and the three fields in the qDFIHC analysis. A significant correlation was observed in each of the three proteins (Spearman's rank correlation coefficient test).

a)

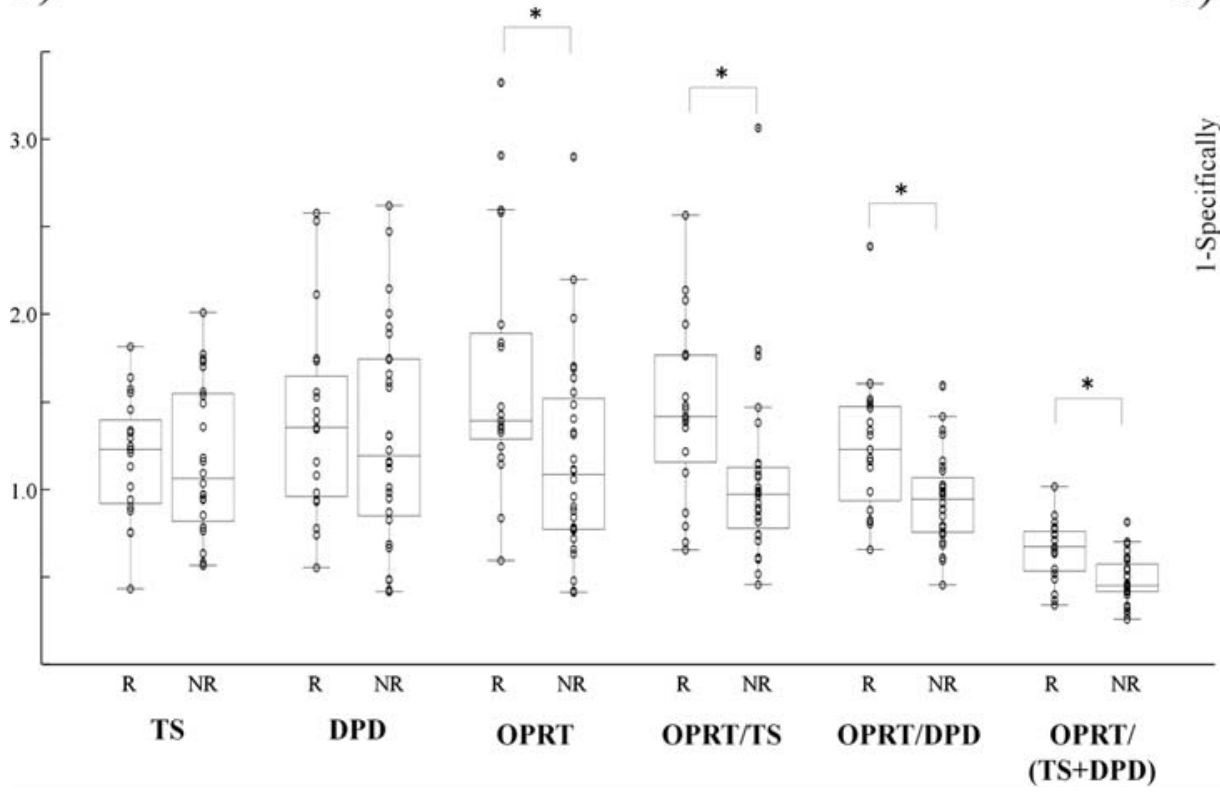

b)

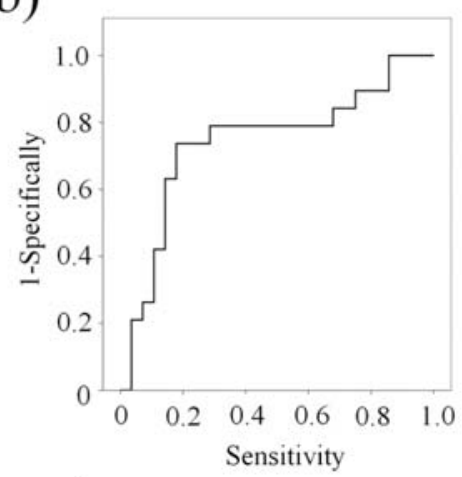

c)

\begin{tabular}{lc}
\hline & Cut-off value \\
\hline OPRT & 1.2 \\
OPRT/TS & 1.2 \\
OPRT/DPD & 1.1 \\
OPRT/TS+DPD) & 0.6 \\
\hline
\end{tabular}

Figure 2. (a) The expression level of the target proteins and the protein ratios assessed by qDFIHC in 47 cases. The comparison between the responders and non-responders is as follows: responders; TS 1.18 \pm 0.35 , DPD 1.39 \pm 0.57 , OPRT 1.67 \pm 0.72 , OPRT/TS 1.45 \pm 0.51, OPRT/DPD 1.25 \pm 0.40, OPRT/(TS+DPD) $0.65 \pm 0.18$, non-responders; TS $1.16 \pm 0.44$, DPD 1.30 \pm 0.61 , OPRT $1.17 \pm 0.58$. OPRT/TS $1.06 \pm 0.51$, OPRT/DPD 0.95 \pm 0.26, OPRT/(DPD+TS) 0.48 \pm 0.13 . A significant difference was observed in OPRT $(\mathrm{P}=0.012)^{*}, \mathrm{OPRT} / \mathrm{TS}(\mathrm{P}=0.005)^{*}, \mathrm{OPRT} / \mathrm{DPD}(\mathrm{P}=0.006)^{*}$ and $\mathrm{OPRT} /(\mathrm{TS}+\mathrm{DPD} ; \mathrm{P}=0.002)^{*}(\mathrm{Mann}-\mathrm{Whitney}$ $\mathrm{U}$ test). "Statistical significance. (b) Receiver operating characteristic (ROC) curve in OPRT/TS is shown. (c) The cut-off values were determined to be 1.2 , 1.2, 1.1 and 0.6 in OPRT, OPRT/TS, OPRT/DPD and OPRT/(TS+DPD) using each ROC curve, respectively. Sensitivity and specificity at each cut-off value is detailed in Table II. 
Table II. Association with cut-off value and chemotherapeutic response in OPRT expression, OPRT/TS, OPRT/DPD and $\mathrm{OPRT} /(\mathrm{TS}+\mathrm{DPD})$ ratio.

\begin{tabular}{|c|c|c|c|c|c|c|c|c|}
\hline & Cut-off value & $\mathbf{R}$ & NR & P-value & Sensitivity (\%) & Specificity (\%) & Accuracy (\%) & $\operatorname{tRR}(\%)$ \\
\hline \multirow[t]{2}{*}{ OPRT } & $\geq 1.2$ & 15 & 11 & $0.009^{\mathrm{a}}$ & 78.9 & 60.7 & 68.1 & 57.7 \\
\hline & $<1.2$ & 4 & 17 & & & & & \\
\hline \multirow[t]{2}{*}{ OPRT/TS } & $\geq 1.2$ & 14 & 5 & $<0.001^{\mathrm{a}}$ & 73.7 & 82.1 & 78.7 & 73.7 \\
\hline & $<1.2$ & 5 & 23 & & & & & \\
\hline \multirow[t]{2}{*}{ OPRT/DPD } & $\geq 1.1$ & 13 & 7 & $0.006^{\mathrm{a}}$ & 68.4 & 75 & 72.3 & 65.0 \\
\hline & $<1.1$ & 6 & 21 & & & & & \\
\hline \multirow[t]{2}{*}{ OPRT/(TS+DPD) } & $\geq 0.6$ & 13 & 6 & $0.002^{\mathrm{a}}$ & 68.4 & 78.6 & 74.5 & 68.4 \\
\hline & $<0.6$ & 6 & 22 & & & & & \\
\hline
\end{tabular}

$\mathrm{R}$, responder; NR, non-responder; tRR, tailored response rate; the response rate was calculated only in the patients belonging to high group of the cut-off values. ${ }^{a} \chi 2$ test. Tailored response rate (tRR) was also determined as RR was calculated only in the patients belonging to high group of OPRT, OPRT/TS, OPRT/DPD or OPRT/(TS+DPD).

Table III. Comparison of OPRT/TS ratio with cut-off value and chemotherapeutic response in the 17 patients who underwent the subsequent gastrectomy.

\begin{tabular}{ccccccc}
\hline Case & OPRT/TS & Cut-off (1.2) & Clinical stage & Macroscopic type & Clinical response & Pathological response \\
\hline 1 & 1.76 & High & IV & 2 & CR & pCR (grade 3) \\
2 & 1.405 & High & IV & 3 & PR & pPR (grade 1b) \\
3 & 1.77 & High & IV & 4 & PR & pNC (grade 1a) \\
4 & 1.217 & High & IV & 4 & PR & pNC (grade 1a) \\
5 & 1.083 & Low & IIIb & 4 & NC & pNC (grade 1a) \\
6 & 0.456 & Low & Ib & 4 & NC & pNC (grade 0) \\
7 & 0.74 & Low & Ib & 4 & NC & pNC (grade 1a) \\
8 & 0.814 & Low & IIIb & 3 & NC & pNC (grade 1a) \\
9 & 0.88 & Low & IV & 4 & NC & pNC (grade 1a) \\
10 & 1.013 & Low & Ib & 4 & NC & pNC (grade 0) \\
11 & 0.842 & Low & IIIa & 4 & NC & pNC (grade 1a) \\
12 & 0.517 & Low & IIIb & 4 & NC & pNC (grade 1a) \\
13 & 0.894 & Low & IIIb & 3 & PR (grade 0) \\
14 & 2.567 & High & IIIa & 3 & NC & pPR (grade 2) \\
15 & 0.602 & Low & IIIb & 3 & CR & pCR (grade 1b) \\
16 & 2.137 & High & IV & 3 & PR & pPR (grade 2) \\
17 & 1.463 & High & IV & 4 & & \\
\hline
\end{tabular}

pPR (grade 2). CR, complete response; PR, partial response; NC, no change.

was calculated only in the patients belonging to the high group of OPRT, OPRT/TS, OPRT/DPD or OPRT/(TS+DPD). OPRT/TS showed the highest tRR (73.7\%; Table II).
qDFIHC and pathological analysis in gastrectomy cases. The pathological as well as the clinical responses to S-1 therapy was further correlated with OPRT, OPRT/TS, OPRT/DPD or OPRT/(TS+DPD) in 17 gastrectomy cases. There was a 

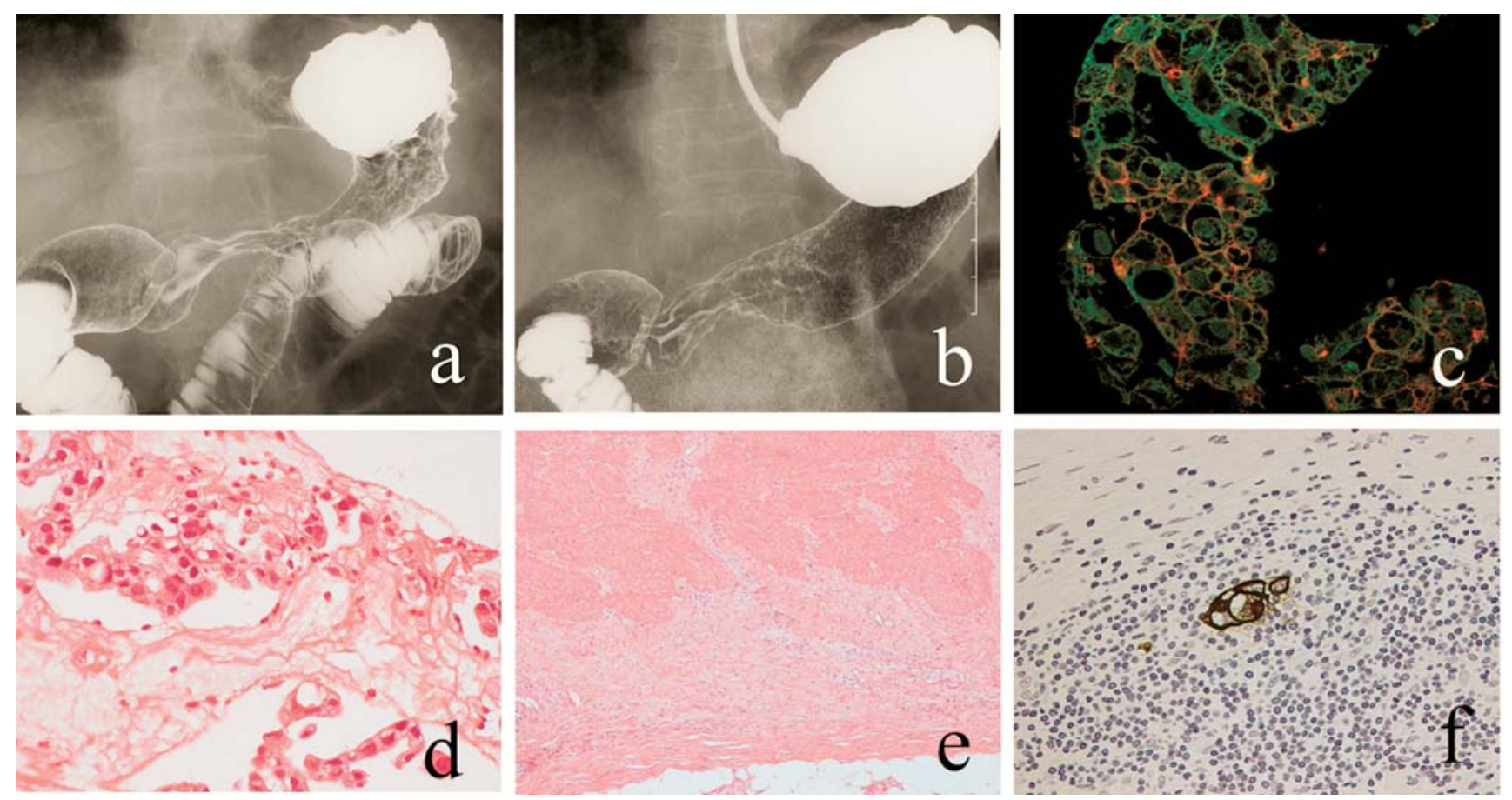

Figure 3. Case presentation (no. 17 in Table III); the evaluated OPRT/TS was 1.463 and this case was expected to be a responder. A gastrectomy was performed after two cycles of S-1 plus cisplatin chemotherapy. (a) The X-ray image before chemotherapy; severe stenosis of gastric lumen suggesting scirrhous gastric cancer was observed. (b) The X-ray image after chemotherapy; the stenosis of gastric lumen was significantly improved. (c) qDFIHC image of biopsy specimen (original magnification x400). (d) The biopsy specimen before chemotherapy. The pathological diagnosis was poorly differentiated adenocarcinoma (HE, original magnification x200). (e) Massive fibrosis reaching the subserosa was observed in the resected stomach whereas no cancer cell was detected in HE staining (original magnification x20). (f) The small cluster of cancer cells was detected in the immunohistochemistry of cytokeratin (original magnification x200). The pathologic chemotherapeutic effect was assessed as grade 2.

statistically significant correlation between the clinical response and all the 4 markers: OPRT $(\mathrm{P}=0.01)$, OPRT/TS $(\mathrm{P}<0.001)$, OPRT/DPD ( $\mathrm{P}=0.003)$, OPRT/(TS+DPD; $\mathrm{P}=0.001)$, in which OPRT/TS showed the strongest correlation in 17 gastrectomy cases (data not shown). Table III summarizes the comparison between the clinical as well as pathological response to NAC by $\mathrm{S}-1$ and the corresponding OPRT/TS values. No PD case was observed in the gastrectomy group. All 7 responders showed a high OPRT/TS, whereas 10 of non-responder showed a low OPRT/TS, resulting in $100 \%$ of tRR. Two patients with a pathological NC (case no. 3 and 4), assessed as non-responders, exhibited a high OPRT/TS. One patient diagnosed as a responder with a pathological PR (no. 15) conversely expressed a low OPRT/TS. The resultant tRR in the pathological response decreased to $71.4 \%$, lower than that in the clinical response (Table III). Fig. 3 demonstrate several images in case no. 17 with a high OPRT/TS.

OPRT expression in the qDFIHC system is shown in Fig. 3c, in which both the fluorescent staining by OPRT and B-actin antibody were merged. NAC by $\mathrm{S}-1$ to this patient resulted in a PR (Fig. 3a and b) and pPR (Fig. 3d-f) in the clinical, and pathological response, respectively.

\section{Discussion}

The Gastrointestinal Oncology Study Group in Japan and the Japan Clinical Oncology Group (GIOSG/JCOG) have conducted several clinical trials to establish a standard chemotherapy for unresectable or recurrent gastric cancer
$(4,5)$. Recent phase III studies such as JCOG 9912 and SPIRITS trial have shown the large impact of S-1 treatment. S-1 based chemotherapy (with or without cisplatin) is considered to be the standard regimen for the treatment of unresectable or recurrent gastric cancer. However, the response rate (RR) to S-1 based chemotherapy is still around $30-40 \%$ (4,5). On the other hand, pre-operative chemotherapy, or 'neoadjuvant chemotherapy', has been applied to resectable gastric cancer, in order to increase the rate of R0 resections (25). In particular, scirrhous gastric cancer shows the poorest prognosis among all gastric cancer patients, even following a gastric resection and D2 lymph node dissection $(6,7)$. In a clinical trial NAC was administered to patients with this type of gastric cancer (6). However, the therapeutic benefits of the neoadjuvant treatment on the postoperative survival still remain controversial (6). If some molecular markers predict S-1 efficacy, S-1 therapy for unresectable as well as resectable gastric cancer could be tailored to the prospective patients, and would also help to prolong survival.

TS, DPD and OPRT have been highlighted as S-1 predictor because of their essential role in 5-FU metabolism (8). Various studies analyzed the TS, DPD and OPRT expression in gastric cancer in comparison to the response to 5-FU or S-1 therapy (9-12,14-18). Ichikawa et al (12) reported the ratio of OPRT/DPD, as assessed by qRT-PCR to significantly correlate with the responsiveness of 5-FU in colorectal cancer. They also reported the combination of OPRT and TS expression to significantly correlate with the responsiveness to S-1 in metastatic gastric cancer. To date, either independent 
or combined TS, DPD, OPRT expressions are widely accepted as a predictive marker of 5-FU. However, the previous studies analyzed TS, DPD and OPRT expression using surgically resected tissue specimens. It is desirable that expression of the makers should be estimated in biopsy specimens prior to administering chemotherapy.

A qDFIHC system was recently developed and used to quantitatively estimate TS, DPD and OPRT expression even in the formalin-fixed biopsy specimens (13). The method in qDFIHC analysis is not complicated, because the digital images of fluorescent signals from TS, DPD, OPRT and an internal marker $\beta$-actin were automatically analyzed as described in Materials and methods. In fact, qDFIHC is capable of quantifying TS, DPD and OPRT expression within 3 days after an endoscopic biopsy. A previous study used this newly developed technology to address the relationship between TS, DPD, OPRT and the clinical response to S-1 treatment. However, the pilot study analyzed only 17 cases, which is too small a number of patients to isolate the reliable determinants predicting S-1 efficacy. Furthermore, it did not indicate whether consistent data are acquired in the same sample due to possible tumor heterogeneity $(26,27)$.

The present study assessed TS, DPD and OPRT using the qDFIHC system in 47 biopsy specimens from advanced gastric cancer patients. TS, DPD and OPRT expression were investigated in three fields from 30 samples and then were compared with those in a single field (Fig. 1). The results showed a significant correlation between both evaluations, thus suggesting the evaluation at a single field to be sufficient to determine the protein expression by the qDFIHC system. In comparison to the clinical response to S-1 therapy, the expression values of OPRT, OPRT/TS, OPRT/DPD and $\mathrm{OPRT} /(\mathrm{TS}+\mathrm{DPD})$ revealed a significant correlation in the 47 cases (Fig. 2). The cut-off value distinguishing a clinical response was appropriately determined according to the ROC curve in the 4 factors and subjected to further analysis (Fig. 2). As a result, OPRT/TS with a cut-off value of 1.2, revealed the highest sensitivity, specificity and accuracy in predicting the clinical response (Table II). Moreover, the tRR, which was the RR only in the patients with high OPRT/TS increased up to $73.7 \%$, in comparison to $40.4 \%$ of the overall RR (Table II). OPRT/TS correlated with the pathological response as well as the clinical response in the 17 gastrectomy cases. The result showed that OPRT/TS completely distinguished non-responder from responder with $100 \%$ of tRR in the clinical response (Table III). OPRT/TS also exhibited the differential power in the pathological response with $71.4 \%$ tRR (Table III). As shown in Fig. 3, one case of scirrhous gastric cancer with a high OPRT/TS (OPRT/TS value: 1.463 > cut-off value: 1.2 ) showed a PR and $\mathrm{pPR}$ after S-1 neo-adjuvant therapy. It was therefore strongly suggested that NAC, followed by surgery should be tailored to such patients.

In conclusion, the TS and OPRT expression in gastric cancer biopsy specimen can be quantitatively assessed in just one field using the qDFIHC system. A possible responder could therefore be selected according to the OPRT/TS ratio before the start of S-1 neoadjuvant chemotherapy. A clinical study using a larger number of patients should be prospectively conducted in the future.

\section{References}

1. Parkin DM, Bray F, Ferlay $\mathrm{J}$ and Pisani P: Global cancer statistics, 2002. CA Cancer J Clin 55: 74-108, 2005.

2. Shirasaka T, Shimamato Y, Ohshimo H, et al: Development of a novel form of an oral 5-fluorouracil derivative (S-1) directed to the potentiation of the tumor selective cytotoxicity of 5fluorouracil by two biochemical modulators. Anticancer Drugs 7: 548-557, 1996.

3. Shirasaka T, Shimamoto Y and Fukushima M: Inhibition by oxonic acid of gastrointestinal toxicity of 5-fluorouracil without loss of its antitumor activity in rats. Cancer Res 53: 4004-4009, 1993.

4. Boku N: Chemotherapy for metastatic gastric cancer in Japan. Int J Clin Oncol 13: 483-487, 2008.

5. Koizumi W, Narahara H, Hara T, et al: S-1 plus cisplatin versus S-1 alone for first-line treatment of advanced gastric cancer (spirits trial): a phase III trial. Lancet Oncol 9: 215-221, 2008.

6. Kinoshita T, Sasako M, Sano T, et al: Phase II trial of S-1 for neoadjuvant chemotherapy against scirrhous gastric cancer (JCOG 0002). Gastric Cancer 12: 37-42, 2009.

7. Hartgrink HH: Improving outcome for scirrhous gastric cancer. Gastric Cancer 12: 3-5, 2009.

8. Longley DB, Harkin DP and Johnston PG: 5-fluorouracil: mechanisms of action and clinical strategies. Nat Rev Cancer 3: 330-338, 2003

9. Ichikawa W, Takahashi T, Suto K, et al: Thymidylate synthase and dihydropyrimidine dehydrogenase gene expression in relation to differentiation of gastric cancer. Int J Cancer 112: 967-973, 2004

10. Ichikawa W, Takahashi T, Suto K, et al: Simple combinations of 5-FU pathway genes predict the outcome of metastatic gastric cancer patients treated by S-1. Int J Cancer 119: 1927-1933, 2006.

11. Ichikawa W, Takahashi T, Suto K, et al: Thymidylate synthase predictive power is overcome by irinotecan combination therapy with S-1 for gastric cancer. Br J Cancer 91: 1245-1250, 2004.

12. Ichikawa W, Uetake H, Shirota Y, et al: Combination of dihydropyrimidine dehydrogenase and thymidylate synthase gene expressions in primary tumors as predictive parameters for the efficacy of fluoropyrimidine-based chemotherapy for metastatic colorectal cancer. Clin Cancer Res 9: 786-791, 2003.

13. Kai K, Kitajima Y, Hiraki M, et al: Quantitative doublefluorescence immunohistochemistry (qDFIHC), a novel technology to assess protein expression: a pilot study analyzing 5-FU sensitive markers thymidylate synthase, dihydropyrimidine dehydrogenase and orotate phosphoribosyl transferases in gastric cancer tissue specimens. Cancer Lett 258: 45-54, 2007.

14. Johnston PG, Lenz HJ, Leichman CG, et al: Thymidylate synthase gene and protein expression correlate and are associated with response to 5-fluorouracil in human colorectal and gastric tumors. Cancer Res 55: 1407-1412, 1995

15. Matsuyama R, Togo S, Shimizu D, et al: Predicting 5-fluorouracil chemosensitivity of liver metastases from colorectal cancer using primary tumor specimens: Three-gene expression model predicts clinical response. Int J Cancer 119: 406-413, 2006.

16. Miyazaki K, Shibahara T, Sato D, et al: Influence of chemotherapeutic agents and cytokines on the expression of 5fluorouracil-associated enzymes in human colon cancer cell lines. J Gastroenterol 41: 140-150, 2006.

17. Salonga D, Danenberg KD, Johnson M, et al: Colorectal tumors responding to 5-fluorouracil have low gene expression levels of dihydropyrimidine dehydrogenase, thymidylate synthase, and thymidine phosphorylase. Clin Cancer Res 6: 1322-1327, 2000.

18. Sato K, Kitajima Y, Miyoshi A, Koga Y and Miyazaki K: Deficient expression of the DPD gene is caused by epigenetic modification in biliary tract cancer cells, and induces high sensitivity to 5-FU treatment. Int J Oncol 29: 429-435, 2006.

19. Sakurai Y, Sakamoto K, Sugimoto Y, et al: Orotate phosphoribosyltransferase levels measured by a newly established enzyme-linked immunosorbent assay in gastric carcinoma. Cancer Sci 97: 492-498, 2006.

20. Tsuchida M, Yamato Y, Hashimoto T, et al: Expression of 5fluorouracil-related enzymes in lung cancer: ELISA characterizes enzyme activity and messenger RNA expression. Oncol Rep 21: 1037-1043, 2009.

21. Sobin LH: TNM, sixth edition: new developments in general concepts and rules. Semin Surg Oncol 21: 19-22, 2003. 
22. Sakata Y, Ohtsu A, Horikoshi N, Sugimachi K, Mitachi Y and Taguchi T: Late phase II study of novel oral fluoropyrimidine anticancer drug S-1 ( $1 \mathrm{~m}$ tegafur- $0.4 \mathrm{~m}$ gimestat- $1 \mathrm{~m}$ otastat potassium) in advanced gastric cancer patients. Eur J Cancer 34: 1715-1720, 1998.

23. Japanese classification of gastric carcinoma - 2nd English edition-response assessment of chemotherapy and radiotherapy for gastric carcinoma: clinical criteria. Gastric Cancer 4: 1-8, 2001.

24. Muller G: [General guidelines for surgery and pathology of the Japanese Research Society for Gastric Cancer]. Chirurg 56: $539-552,1985$
25. D'Ugo D, Rausei S, Biondi A and Persiani R: Preoperative treatment and surgery in gastric cancer: friends or foes? Lancet Oncol 10: 191-195, 2009.

26. Mercer SJ, Somers SS, Knight LA, et al: Heterogeneity of chemosensitivity of esophageal and gastric carcinoma. Anticancer Drugs 14: 397-403, 2003.

27. Schlag P and Schreml W: Heterogeneity in growth pattern and drug sensitivity of primary tumor and metastases in the human tumor colony-forming assay. Cancer Res 42: 4086-4089, 1982. 